\title{
Treinamento com pesos, modelo de Programa Sistematizado para a Terceira Idade
}

Weight training, Systematized Program Model for the Elderly

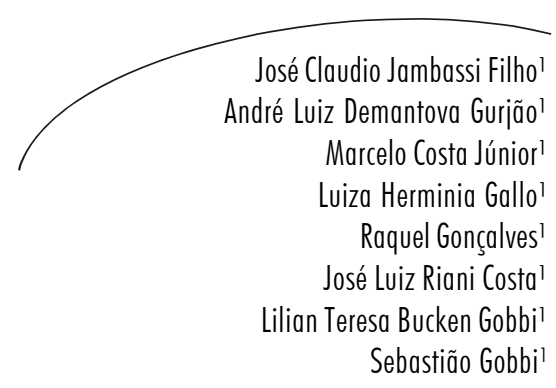

Resumo

Evidências têm demonstrado que a manutenção de um estilo de vida fisicamente ativo pode atenuar as perdas associadas ao processo de envelhecimento. Dentre as práticas recomendadas para a população idosa, o treinamento com pesos (TP) tem recebido destaque, revelando benefícios na realização das atividades da vida diária e na qualidade de vida, entre outros. Assim, programas para pessoas idosas devem descrever suas experiências na tentativa de impulsionar e aperfeiçoar as atividades relacionadas com TP. Desta forma, o presente trabalho tem como objetivo descrever o funcionamento do TP, que é umas das modalidades do Programa de Atividade Física para a Terceira Idade (PROFIT). Atualmente, a modalidade TP conta com 45 participantes, sendo que $80 \%$ são mulheres e $20 \%$ homens. A maior parte dos participantes (84\%) possui idade igual ou superior a 60 anos, ao passo que $16 \%$ possuem idade entre 54 e 59 anos. As aulas são ministradas três vezes por semana, em dias não consecutivos, com duração de 45 minutos. Os participantes realizam oito exercícios alternados por segmentos corporais, sendo realizadas três séries em cada exercício. Neste sentido, a modalidade TP busca proporcionar melhorias na qualidade de vida da população idosa, bem como auxiliar na formação de graduandos e pós-graduandos e, consequentemente, aprofundar o entendimento da atividade física em idosos.

\section{Abstract}

Evidence has shown that maintaining a healthy physically active lifestyle can reduce the losses associated with the aging process. Among the recommended exercises for elderly people, weight training (WT) has received highlights, revealing benefits related to their performance of daily living activities and quality of life, among others. Thus, programs for elderly people should describe their experiences in an attempt to boost and to refine the activities related to WT. Thus

Palavras-chave: Estilo de vida. Saúde do idoso. Atividade Motora. Envelhecimento. Promoção da saúde. Treinamento de resistência.

\footnotetext{
Universidade Estadual Paulista-Unesp. Instituto de Biociência. Departamento de Educação Física.
} Laboratório de Atividade Física e Envelhecimento (LAFE). Rio Claro, SP, Brasil. 
this paper aims to describe the operation of the WT, which is one of the modes of the Physical Activity Program for Third Age (PROFIT). Currently the WT program has 45 participants, of which $80 \%$ are women and $20 \%$ are men. Most participants (84\%) are aged 60 years, whereas $16 \%$ are aged between 54 and 59 years. Classes are held three times a week on nonconsecutive days, lasting 45 minutes. The participants perform eight exercises by alternating body segments, and are held three sets of each exercise. Accordingly, the WT program seeks to provide improvements in quality of life of the elderly population, as well as assist in undergraduate and graduate students formation and thus deepen the understanding of the effects of physical activity in older people.
Key words: Life Style. Health of the Elderly. Motor Activity. Aging. Health Promotion. Resistance training.

\section{INTRODUÇÃO}

Nas últimas décadas, o número de adultos idosos está aumentando de forma considerável no Brasil e no mundo. Em países desenvolvidos, a expectativa de vida tem aumentado linearmente e estima-se que metade dos indivíduos nascidos a partir de 2000 comemorará 100 anos de idade. ${ }^{1}$ No Brasil, estima-se que para o ano de 2050 haverá aproximadamente 64 milhões de idosos, o que corresponderá a cerca de $30 \%$ da população. ${ }^{2} \mathrm{Em}$ consequência, o aumento da expectativa de vida da população está acompanhado de perdas fisiológicas e estruturais, podendo ocasionar redução da qualidade de vida. ${ }^{3}$

Por outro lado, há evidências de que a manutenção de um estilo de vida fisicamente ativo pode atenuar as perdas associadas ao processo de envelhecimento, ${ }^{4}$ além de aumentar a expectativa de vida. ${ }^{5}$ Adicionalmente, idosos participantes de programas sistematizados de atividades físicas apresentam maiores níveis de atividade física do que idosas não participantes. ${ }^{6}$ Atualmente, a prática de treinamento com pesos (TP) tem sido recomendada para a população idosa, apresentando diversos benefícios, tais como: manutenção e desenvolvimento da força muscular, ${ }^{7}$ alterações nos indicadores da composição corporal, ${ }^{8}$ bem como um importante contribuinte para a melhora da funcionalidade e manutenção da independência. ${ }^{9}$

Neste sentido, na perspectiva de proporcionar melhorias na qualidade de vida da população idosa e auxiliar na formação de graduandos e pósgraduandos, diversos programas de atividades físicas foram implantados nas universidades em diferentes regiões de nosso país. ${ }^{10-12}$ Assim, na tentativa de impulsionar a formulação de novos programas, proporcionando o aumento na quantidade de pessoas beneficiadas e melhora do conhecimento sobre a atividade física, torna-se necessário que as experiências vivenciadas por esses programas sejam evidenciadas e relatadas para o escopo cientifico. Desta forma, o presente trabalho tem como objetivo descrever o funcionamento do TP, que é umas das modalidades do Programa de Atividade Física para a Terceira Idade (PROFIT).

\section{VISÃO GERAL DO PROGRAMA}

$\mathrm{Na}$ tentativa de proporcionar benefícios biopsicossociais à população idosa, bem como auxiliar na formação de graduandos e pósgraduandos, em março de 1989, foi criado o PROFIT. O projeto está vinculado ao Departamento de Educação Física (DEF), do Instituto de Biociências da UNESP (IB) - Campus de Rio Claro, e ao Laboratório de Atividade Física e Envelhecimento (LAFE).

Com o intuito de possibilitar a inserção da população idosa à universidade pública, o Núcleo UNESP-UNATI (Universidade Aberta à Terceira Idade) estimula o desenvolvimento de atividades de ensino, pesquisa e extensão ligadas às questões concernentes ao processo de envelhecimento. Por sua vez, o Núcleo UNESP-UNATI está vinculado a Pró-Reitoria de Extensão Universitária (PROEX), que é comprometida com o apoio financeiro para o desenvolvimento das atividades 
de extensão universitária. Adicionalmente, a Fundação para o Desenvolvimento da UNESP (FUNDUNESP) auxilia na interação $\mathrm{da}$ universidade com a sociedade, por meio da intermediação de convênios e projetos, fomento à pesquisa, entre outros apoios.
Desta forma, por meio dos diversos apoios que o PROFIT recebe, sua estrutura vem sendo modificada e consequentemente envolvendo uma maior quantidade de idosos nas diferentes atividades oferecidas, dentre elas o TP. Na figura 1, encontrase descrito o modelo lógico da modalidade TP.

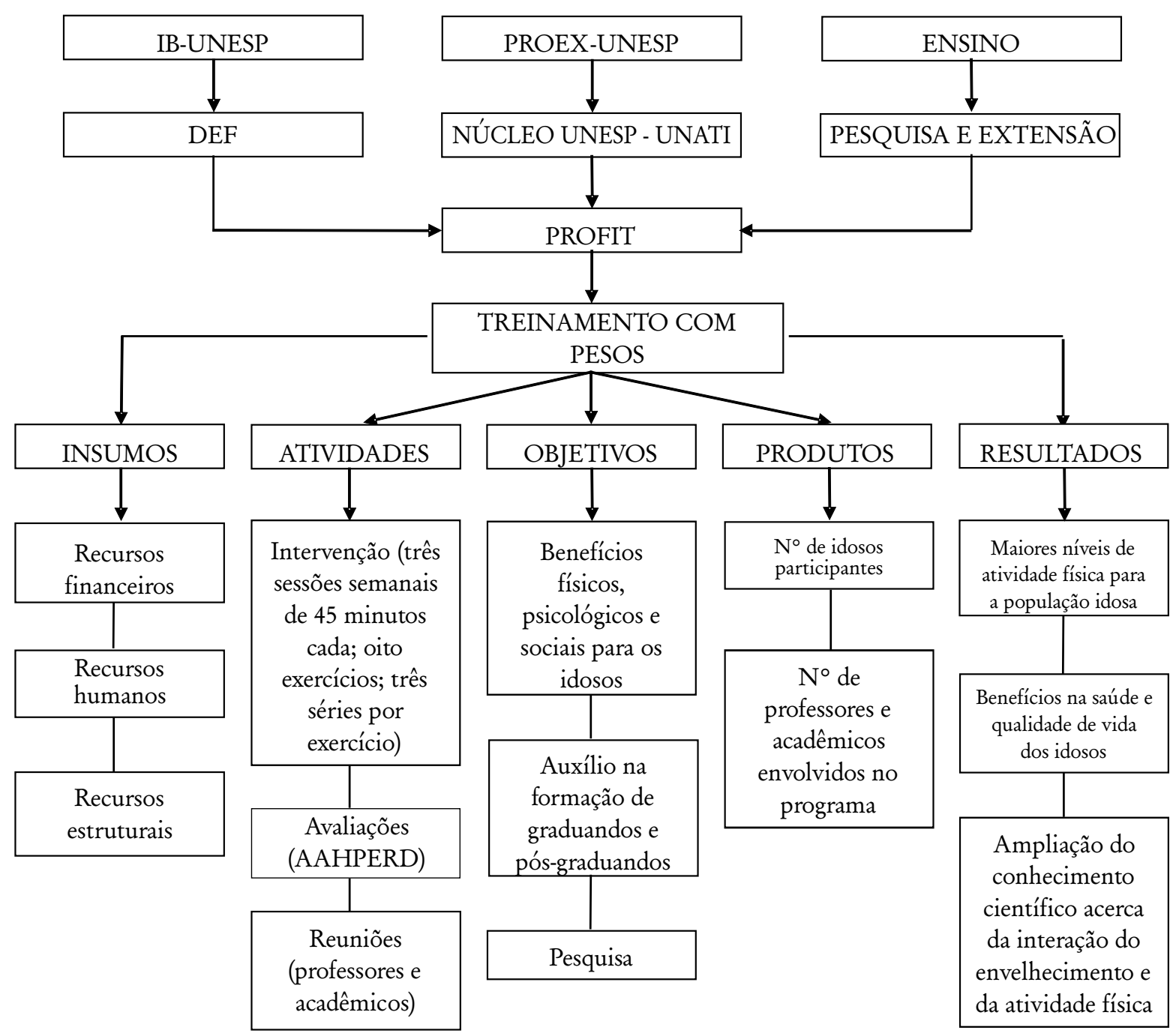

PROEX: Pró-Reitoria de Extensão Universitária; UNESP-UNATI: Universidade Estadual Paulista - Universidade Aberta à Terceira Idade; PROFIT: Programa de Atividade Física para a Terceira Idade; IB-UNESP: Instituto de Biociências da Universidade Estadual Paulista; DEF: Departamento de Educação Física

Figura 1 - Modelo lógico da modalidade treinamento com pesos. 


\section{TREINAMENTO COM PESOS}

Inicialmente, em cada dia de atividade do projeto era realizada uma modalidade, dentre elas o TP. Somente a partir do ano de 2003, foi criada uma turma que realizava apenas a modalidade TP. Atualmente a modalidade TP conta com 45 participantes, sendo que $80 \%$ são mulheres $(65,2 \pm 6,2$ anos; $73,1 \pm 11,8 \mathrm{Kg} ; 1,58$ $\left.\pm 0,06 \mathrm{~cm} ; 29,1 \pm 4,5 \mathrm{Kg} / \mathrm{m}^{2}\right)$ e $20 \%$ homens
$(67,8 \pm 5$ anos; $85,2 \pm 17,3 \mathrm{Kg} ; 1,71 \pm 0,05$ $\left.\mathrm{cm} ; 29,1 \pm 4,9 \mathrm{Kg} / \mathrm{m}^{2}\right)$. A maior parte dos participantes $(84 \%)$ possui idade igual ou superior a 60 anos $(67,3 \pm 5,1$ anos; 76,3 \pm $\left.13,4 \mathrm{Kg} ; 1,61 \pm 0,08 \mathrm{~cm} ; 29,3 \pm 4,5 \mathrm{Kg} / \mathrm{m}^{2}\right)$, ao passo que $16 \%$ possuem idade entre 54 e 59 anos $(57,1 \pm 1,8$ anos; $69,2 \pm 13 \mathrm{Kg} ; 1,57 \pm$ $0,04 \mathrm{~cm} ; 27,9 \pm 5 \mathrm{Kg} / \mathrm{m}^{2}$ ). A figura 2 apresenta a quantidade de participantes (idosos e estagiários) da modalidade TP, a partir de 2003.

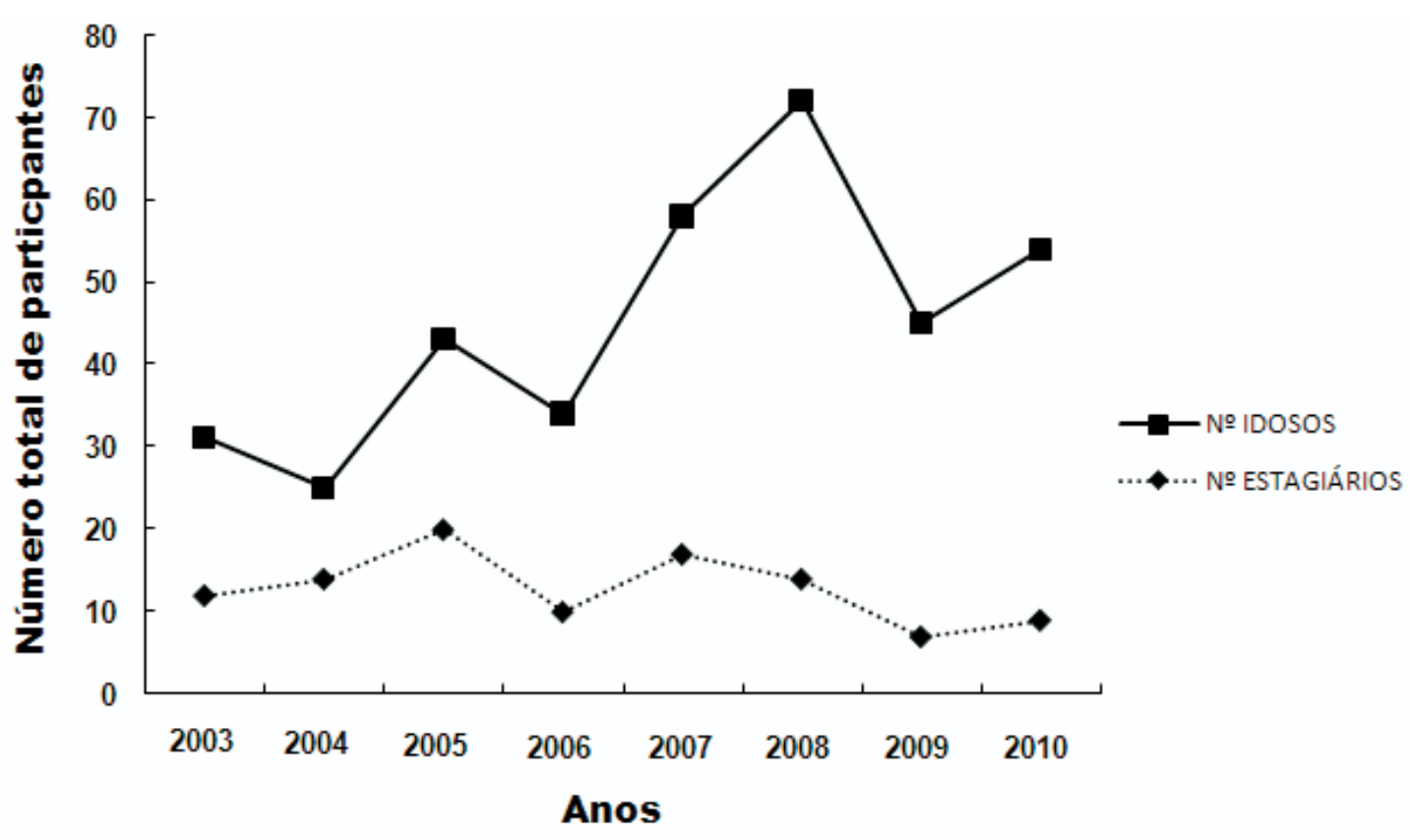

Figura 2 - Quantidade anual de participantes (idosos e estagiários) da modalidade TP, a partir de 2003.

$\mathrm{O}$ aumento no número de participantes a partir do ano de 2006 pode ser explicado pela criação de uma nova turma. Por outro lado, a redução no número de integrantes no ano de 2009, se deve ao fato de ser adotada frequência semestral mínima de $90 \%$.

$\mathrm{Na}$ tentativa de homogeneizar os estudos realizados no programa, atualmente estão sendo recrutadas somente participantes do sexo feminino. Os homens que permanecem no projeto são aqueles que já estavam engajados na implantação deste critério. Toda idosa interessada em adentrar na modalidade TP preenche um cadastro com os dados pessoais e permanece na lista de espera. Existindo a vaga, a interessada é convocada e deve apresentar um relatório de avaliação médica indicando não apresentar restrições clínicas de ordem cardiovascular, muscular, articular, óssea ou neurológica para a prática da atividade.

O projeto é realizado durante o ano, com dois períodos de férias (julho; dezembro e janeiro). Durante o período de treinamento, as aulas são ministradas três vezes por semana, em dias não consecutivos (segunda, quarta e sexta-feira), com duração de 45 minutos. Todas as sessões de 
treinamento são orientadas por profissionais de Educação Física (pós-graduados e graduados) e estagiários (graduandos).

Com objetivo de fornecer subsídios para o planejamento mensal das aulas e para analisar a efetividade do treinamento em diferentes componentes da capacidade funcional (flexibilidade, coordenação, agilidade e equilíbrio dinâmico, resistência de força e capacidade aeróbia), a bateria completa de testes da American Alliance for Health, Physical Education, Recreation and Dance (AAHPERD) ${ }^{13,14}$ é sistematicamente empregada no início do projeto, no retorno das férias de julho e ao seu final (em dezembro). Em adição, para avaliar a necessidade de modificações na especificidade do treinamento ou nos procedimentos operacionais adotados para o desenvolvimento das aulas, reuniões mensais são realizadas com os coordenadores e estagiários $\mathrm{da}$ modalidade. Dessa forma, é possível direcionar regularmente o programa de TP para o desenvolvimento de um ou mais componentes da capacidade funcional, especialmente para os componentes que se encontram classificados abaixo do percentil 50 (regular) para os valores normativos associados à idade e gênero. ${ }^{14-16}$

Os resultados obtidos pela bateria de testes são apresentados individualmente aos participantes por meio de relatórios impressos (de fácil compreensão) do desempenho de cada componente avaliado e por esclarecimentos verbais. Esse procedimento tem se mostrado útil como fator motivacional para a continuidade dos participantes no projeto.

Para ilustrar o comportamento da capacidade funcional no decorrer do tempo, a figura 3 apresenta o Índice de Aptidão Funcional Geral (IAFG) da última avaliação anual dos últimos dois anos até o presente (avaliação de julho). O IAFG é determinado pela soma dos escores-percentis dos cinco testes da bateria da AAHPERD e parecer estar se mantendo estável ao longo do tempo.

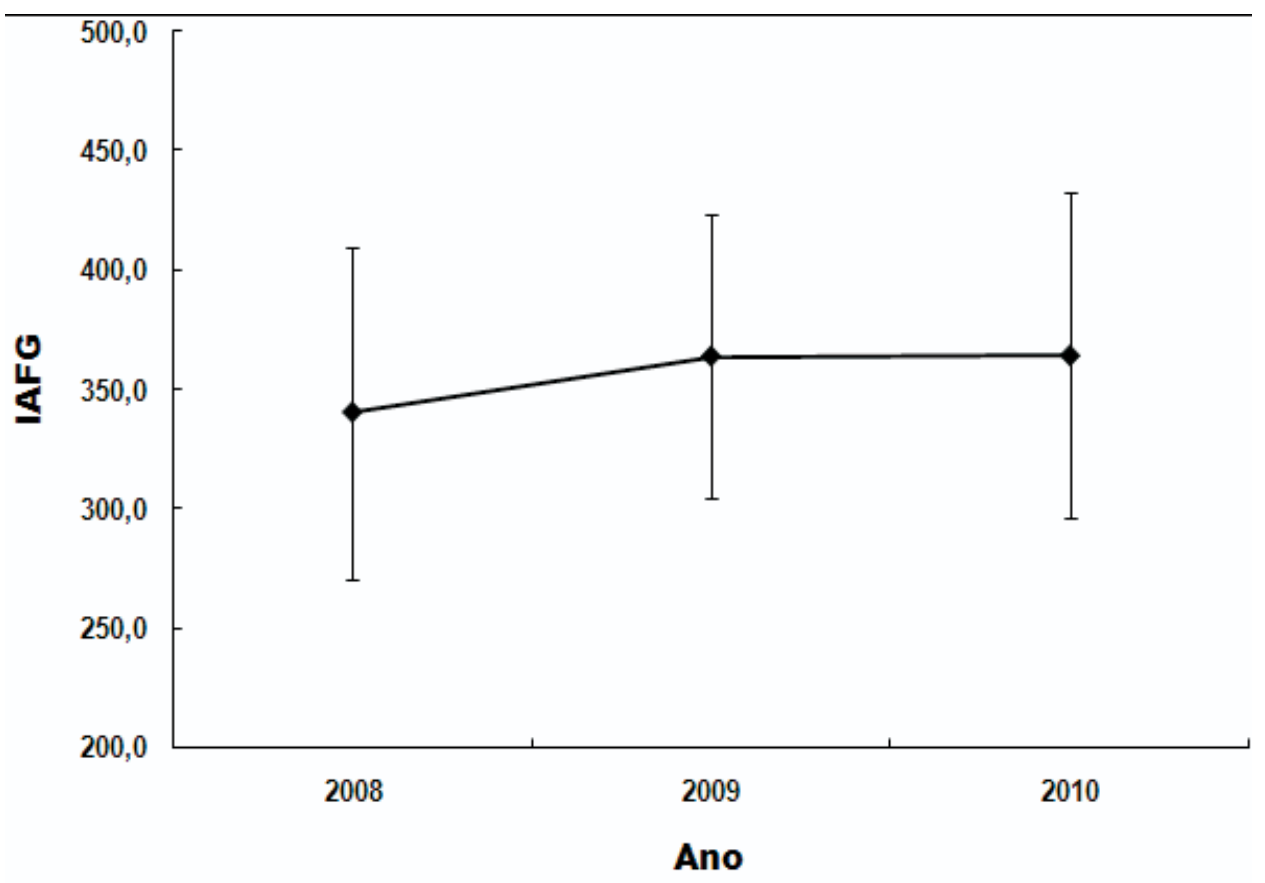

Figura 3 - Índice de aptidão funcional geral determinado pela soma dos escores-percentis dos cinco testes da bateria da American Alliance for Health, Physical Education, Recreation and Dance. 
$\mathrm{O}$ protocolo de treinamento segue as atuais recomendações do Colégio Americano de Medicina do Esporte (ACSM, 2009) ${ }^{17}$ e seu delineamento é realizado por meio da manipulação das diferentes variáveis do TP, especificamente: intensidade, volume, velocidade de contração e duração do intervalo de recuperação entre séries e exercícios. As participantes realizam oito exercícios alternados por segmentos corporais. A carga varia conforme o objetivo do treinamento, sendo determinada por meio do teste de RM, como descrito em estudos prévios. ${ }^{18,19}$ Em geral, as intensidades de treinamento variam entre 10 e 15$\mathrm{RM}$, realizadas em três séries para cada exercício. As cargas são ajustadas para que a fadiga ocorra na última série. Como aquecimento prévio, os participantes realizam uma série de dez repetições a 50\% da carga que será empregada nas três séries subsequentes. Para manter a intensidade do treinamento ao longo do tempo, quando o número de repetições preestabelecido excede duas repetições, incrementos de carga na ordem de $2-10 \%$ são realizadas. Embora a velocidade de execução não seja controlada, as participantes são instruídas a executarem cada repetição em aproximadamente um segundo na fase concêntrica e em aproximadamente dois segundos na fase excêntrica.

Ao considerar que a modalidade de TP do PROFIT também contribui para o desenvolvimento de trabalhos científicos, sempre que um projeto de pesquisa é idealizado, o mesmo é encaminhado previamente ao Comitê de Ética da Universidade. Após sua aprovação, os participantes que atenderem ao convite para participar do estudo recebem informações sobre as finalidades e procedimentos do trabalho e assinam o termo de consentimento livre e esclarecido. Os resultados decorrentes desses trabalhos, que enfocam principalmente a análise do efeito agudo e/ou crônico do TP nas respostas neuromusculares, hemodinâmicas, metabólicas e hormonais de idosos, são apresentados em eventos e revistas cientificas.

\section{DISCUSSÃO}

Embora o aumento da expectativa de vida da população idosa seja um importante indicativo da melhoria da qualidade de vida, o processo de envelhecimento está atrelado a perdas fisiológicas e estruturais, as quais culminan no declínio da capacidade funcional e independência do idoso. ${ }^{20}$

Em contrapartida, evidências têm demonstrado que a prática regular de atividade física é um importante contribuinte na prevenção, proteção e promoção da saúde de pessoas idosas, melhorando assim a qualidade de vida dessas pessoas $^{8}$. Entretanto, para que esses benefícios sejam alcançados, pessoas adultas idosas devem participar de programas sistematizados. ${ }^{21}$

Os estudos que investigaram o processo de adesão e as ações adotadas para aumentar a participação na prática regular de exercícios físicos demonstraram que as intervenções e aconselhamentos tradicionais são válidos em curto prazo, mas são pouco efetivos em alcançar aderência no longo prazo. ${ }^{22} \mathrm{~A}$ maioria dessas iniciativas tem por objetivo ampliar o conhecimento da população sobre a prática e os benefícios do exercício físico regular. ${ }^{22}$ No entanto, segundo Dishman et al., ${ }^{23}$ menos de $5 \%$ da população acredita que um maior conhecimento sobre os benefícios dos exercícios físicos contribuiria para um aumento de sua prática. Desta forma, apesar de o aumento do conhecimento científico ser de suma importância para a implantação de novos programas de exercício físico, outros fatores devem ser levados em consideração.

Neste sentido, o PROFIT adota diferentes estratégias na tentativa de manter as participantes motivadas e permanecerem no programa. Dentre as estratégias adotadas, estão a realização de palestras com diferentes profissionais da saúde e pelos pós-graduandos da UNESP, realização de diversos eventos, tais como: festas juninas, semana do idoso, cafés da manhã, almoços, visitas a asilos, churrascos, dentre outros. Além disso, as participantes são informadas sobre os procedimentos e objetivos adotados dentro do projeto, bem como são fornecidos relatórios do desempenho dos testes e os resultados das pesquisas. 
A manutenção destas pessoas em programas sistematizados de exercício físico é muito importante para redução da prevalência de pessoas sedentárias em todas as regiões do país. Matsudo $\&$ Matsudo ${ }^{24}$ demonstraram o impacto positivo do programa Agita São Paulo na área metropolitana de São Paulo, avaliando os níveis de atividade física em mais de 3.000 indivíduos entre 1999 e 2004 . Os resultados demonstraram redução na taxa de sedentarismo de $15 \%$ para $11 \%$, e de $30 \%$ para $27 \%$ para pessoas irregularmente ativas, sendo que a população ativa aumentou de $55 \%$ para $62 \%$.

Recentemente, Kokobun et al. ${ }^{10}$ relataram a experiência de seis anos de um programa desenvolvido em Unidades Básicas de Saúde no município de Rio Claro-SP. O programa é composto de exercícios cardiorrespiratórios (caminhadas e atividades lúdicas) e neuromotores (força, agilidade, equilíbrio, flexibilidade e coordenação). Nesse período, os autores relataram que os resultados do programa se mostraram efetivos em melhorar o desempenho de alguns componentes da aptidão funcional, metabolismo de lipídios e glicose, estados de ânimo e qualidade de vida relacionada à saúde dos participantes.

O PROFIT conta com o apoio intelectual do Laboratório de Atividade Física e Envelhecimento (LAFE) e Laboratório de Estudos da Postura e Locomoção (LEPLO), para desenvolver diferentes estudos no intuito de melhor compreender os efeitos do TP nos diferentes componentes da capacidade funcional em idosos. Gonçalves et al..$^{25}$ analisaram o efeito de oito semanas de treinamento

\section{REFERÊNCIAS}

1. Christensen $\mathrm{K}$, et al. Ageing population: the challenges ahead. The Lancet 2009;374:1196-1208.

2. Instituto Brasileiro de Geografia e Estatísitica ( IBGE). Séries estatísticas e séries Históricas. São Paulo: IBGE; 2010 [ Acesso 30 Out 2010]. Disponível em: URL: http://www.ibge.gov.br/ series_estatisticas/ subtema.php?idsubtema $=125$.

3. Levinger I, et al. The effect of resistance training on functional capacity and quality of life in com pesos (TP) sobre a flexibilidade de idosos. Os resultados sugeriram que o TP pode contribuir para a manutenção ou mesmo aumento $\mathrm{da}$ flexibilidade em diferentes movimentos e articulações de idosos. Outro estudo realizado no projeto demonstrou que mulheres idosas treinadas apresentam níveis de força muscular nos flexores de cotovelo similares a mulheres jovens. ${ }^{19}$

Neste sentido, diversos têm sido os esforços governamentais e de diferentes entidades (públicas e privadas), na tentativa de promover aumento nos níveis de atividade física da população em geral. Assim, programas de atividades físicas que contribuam para o aumento da qualidade de vida dos idosos, bem como para melhoria dos aspectos socioeconômicos devem ser estimulados em todas as regiões do país.

Por esses motivos, o PROFIT tem sua proposta sustentada no envelhecimento ativo, tentando proporcionar a manutenção e/ou o aumento dos níveis de atividade física da população idosa. Adicionalmente, o PROFIT tem como objetivo contribuir com a ampliação do conhecimento científico acerca da interação do envelhecimento e da atividade física, bem como auxiliar na formação de graduandos e pósgraduandos.

\section{AGRADECIMENTOS}

Os autores deste trabalho agradecem ao CNPq, CAPES, FAPESP, NÚCLEO UNESPUNATI, PROEX, FUNDUNESP, PROFIT e ao LAFE. individuals with high and low numbers of metabolic risk factors. Diabetes Care 2007;30(9):2205-10.

4. Mänty $\mathrm{M}$, et al. Long-term effect of physical activity counseling on mobility limitation among older people: a randomized controlled study. J Gerontol a Biol Sci Med Sci 2009; 64(1):83-9.

5. Matsudo SMM, Marin RV, Matsudo VKR. Atividade física e envelhecimento saudável. Diagn Tratamento 2008;13:142-147. 
6. Gobbi S, Menezes E, Tanaka K. Programas supervisionados de atividades físicas estão associados à maior nível de atividade física comparados com não supervisionados. Rev Bras Ativ Fís Saúde 2007;12(1):3-7.

7. Hunter GR, McCarthy JP, Bamman MM. Effects of resistance training on older adults. Sports Med 2004;34(5):329-48.

8. Wleser M, Haber P. The effects of systematic resistance training in the elderly. Int J Sports Med 2007;28(1):59-65.

9. Hazell T, Kenno K, Jakobi J. Functional benefit of power training for older adults. J Aging Phys Act 2007;15(3):349-59.

10. Kokubun E, et al. Programa de atividade física em unidades básicas de saúde: relato de experiência no município de Rio Claro-SP. Rev Bras Ativ Fís Saúde 2007;12(1):45-53

11. Mazo GZ, et al. Do diagnóstico à ação: grupo de estudos da terceira idade: alternativa para a promoção do envelhecimento ativo. Rev Bras Ativ Fís Saúde 2009;14(1):65-70.

12. Paiva ACS, et al. Dança e envelhecimento: uma parceria em movimento.Rev Bras Ativ Fís Saúde 2010;15(1):70-2.

13. Osness WH, et al. Functional fitness assessment for adults over 60 Years: the American alliance for health, physical education, recreation and dance. New York: Reston;1990.

14. Benedetti TRB, et al. Valores normativos de aptidão funcional em mulheres de 70 a 79 anos. Revista Bras Cineantropom Desempenho Hum 2007,9(1):28-36.

15. Mazo GZ, et al. Valores normativos e aptidão funcional em homens de 60 a 69 anos de idade. Rev Bras Cineantropom Desempenho Hum 2010;12(5):316-323.
16. Zago AS, Gobbi S. Valores normativos da aptidão funcional de mulheres de 60 a 70 anos. R Bras Ci. Mov 2003,11(2):77-86.

17. American college of sports medicine. Position stand: progression models in resistance training for healthy adults. Med Sci Sports Exerc 2009;41(3):687-708.

18. Jambassi Filho JC, et al. O efeito de diferentes intervalos de recuperação entre as séries de treinamento com pesos, na força muscular em mulheres idosas treinadas. Rev Bras Med Esporte 2010;16(2):112-5.

19. Barboza BHV, et al. Declínio relacionado a idade sobre a taxa de desenvolvimento de força e o efeito do treinamento com pesos em idosas. Acta Fisiatr 2009;16(1):4-9.

20. Matsudo SMM, Matsudo VKR, Araújo TL. Perfil do nível de atividade física e capacidade funcional de mulheres maiores de 50 anos de idade de acordo com a idade cronológica. Rev Bras Ativ Fís Saúde 2001;6(1):12-24.

21. American College of Sports Medicine. Position stand: exercise and physical activity for older adults. Med Sci Sports Exerc 2009;41(7):1510-30.

22. Ferreira MS, Najar AL. Programas e campanhas de promoção da atividade física. Cien Saude Colet 2005;10:207-19.

23. Dishman RK, Sallis JF, Orenstein DR. The determinants of physical activity and exercise. Public Health Rep 1985;100(2):158-71.

24. Matsudo SMM, Matsudo VKR. Nutrition and an active life: from knowledge to action. Pan American Health Organization 2005;612:141-159.

25. Gonçalves R, Gurjão ALD, Gobbi S. Efeito de oito semanas do treinamento de força na flexibilidade de idosos. Rev Bras Cineantropom 2007;9(2):145-53. 